\title{
How it outsourcing impacts on value creation of manufacturer?
}

\author{
Li-Shan Chen ${ }^{1}$ and Yen-Ming Tseng ${ }^{2, *}$ \\ ${ }^{1}$ School of Management, Fujian University of Technology, China \\ ${ }^{2}$ Key Laboratory of Big Data Mining and Application/ School of Information Science and Engineering, Fujian University of Technology, \\ China
}

\begin{abstract}
A growing concern among the manufacturers who are actively involved in Information Technology outsourcing is post-contract management and the ensuing development of what many practitioners have coined the 'outsourcing partnership'. Over the past few decades, information technology outsourcing has become a widely used and researched means for manufacturers to enhance their performance. Increasing attention has been paid to building successful partnerships in information systems outsourcing. This study employs strategic social positioning, customer participation, customer orientation, and communication culture to develop research model. The quantitative method will be used to validate the research model and test hypotheses developed. This study uses survey method to collect data, and analysis of the reliability, validity and test the proposed hypotheses by Smart Partial Least Squares. The purpose of this study is to use above four factors to produce value co-creation between outsourcing vendors and manufacturers. The final aim is for the future collaboration. This combination will allow manufacturers and information technology outsourcing vendors to increase their value co-creations in the fields of these factors.
\end{abstract}

\section{Introduction}

Despite the development of IT outsourcing and its pledge to keep costs down, few studies have examined its impact on prices. In our global economy, successful e-commerce enterprises need to consider many challenges: a worldwide convergence of enterprises, voluntary enterprises, whacked data exercises, altering consumers' needs, saved production lifecycle, instant access to information, growing market divergence, heightened levels of in-depth business intelligence, online bargains, enhanced business harmonization and rapid variations in IT. [1-3]

Pagani commences by summarizing the results of a study on value networks and value-creation articles in the literature on information systems, strategic management and operations. In strategic management research, many scholars have researched the notion of value and sustainable competition. [4]

Mol et al. describe a path of composite valueincreasing gradations that ultimately forms an entire value recommendation. In their model, each gradation contributes a special ratio to the entire value creation, while value seizing by contributors relies on participant's relative contracting authority. [5-9]

Woodard et al. define digital enterprise strategies as a planned competition by e-commerce enterprises through distributing digitally enabled products or services [10]. Bharadwaj et al. recognize four key areas that influence our reasoning on digital enterprise strategies and that help create a structure to describe the next generation of perceptions [11]. Oslo recognizes four principal groups of originality: product; process; organizational creation and marketing creation [12]. Nevertheless, most studies concentrate on the influence of personal creative actions in segregating or comprehending technological creation [13-14]. E-commerce enterprises seek a technologybased competing advantage, whereas society, academia and technological departments pursue an opportunity to comprehend the world in a unique manner [15].

To expand our knowledge of the link between outsourcing vendors and manufacturers, we have four main goals. First, we examine whether strategic social positioning positively influences value co-creation. Second, we examine whether customer participation positively influences value co-creation. Third, we examine whether customer orientation positively influences value co-creation. Fourth, we examine whether communication culture positively influences value co-creation.

\section{Theory and hypotheses}

We review the literatures related to the major concepts before developing hypotheses and suggested that strategic social positioning may be positively associated with value co-creation, customer participation may be positively associated with value co-creation, customer orientation may be positively associated with value cocreation, and communication culture may be positively associated with value co-creation. We present an

Corresponding author: swk1200@qq.com 
operational definition of the value co-creation construct, then turn to definitions found in the literature for strategic social positioning, customer participation, customer orientation, and communication culture. The research model of this research is shown in Fig.1.

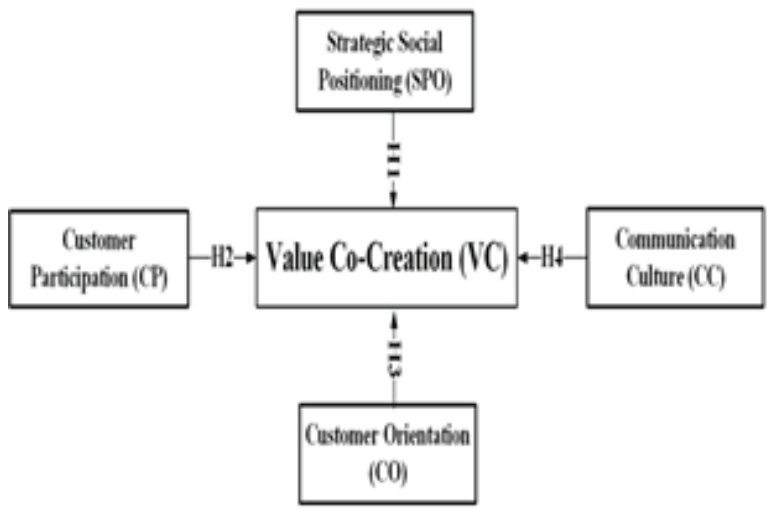

Fig.1. Research Model.

\subsection{Value co-creation}

The value co-creation construct has been introduced by Prahalad and Ramaswamy [16]. They suggest that service values are not created by manufacturers or suppliers alone but are co-created by them together with IT outsourcing vendors. Based on this construct, researchers have devised models to examine how values are co-created. For instance, Maglio and Spohrer use the value co-creation construct in clarifying the essentials of service science, whereas $\mathrm{Wu}$ and $\mathrm{Wu}$ apply it to construct a service process structure. Dong et al. investigate the contextual relation of service failures in a value co-creation situation [17-19].

\subsection{Strategic social positioning}

The positioning determination is frequently a critical strategic determination for e-commerce enterprises because the situation can be foremost in patrons' comprehension and choice determination [20]. Positioning is frequently regarded, erroneously, as a term interchangeable with the psychological concept of standing. While there are similarities between the concepts, there are also major differences [21]. Accordingly, we hypothesize as follows:

H1: Strategic social positioning has a positive impact on value co-creation.

\subsection{Customer participation}

Customer participation may increase communication and relationship building between customers and employees [22-23]. According to health care literature, when patients work with doctors to incorporate their preferences and values, this improves the level of care delivered and encourages more empathetic, honest, and friendly interactions, which produces relational values. On the service provider side, employees may fulfill their social needs for approval when they cocreate services with customers, similar to the way their perceptions of being valued by the organization enable them to satisfy their social needs for approval, affiliation, and esteem [24-26]. Thus, every interaction between employees and customers represents an opportunity to co-create relational values for both parties [27]. Illustrative comments from service employees about camaraderie and social bonding with their customers support this assertion [28]. Based on the statements above, hypothesis 1 is proposed as follows.

$\mathrm{H} 2$ : Customer participation has a positive impact on value co-creation.

\subsection{Customer Orientation}

Customer orientation is based on customer-driven value creation [29-31]. At the firm level, Slater and Narver emphasized that market-oriented firm's place highest priority on the profitable creation, and maintenance of superior customer value. In this study, value creation has been defined as customer's perspective of achieving desired benefits attributable to the salesperson and the company [32]. Saxe and Weitz suggest that customer orientation at an individually employee level is closely related to a 'concern for others' dimension [33]. Therefore, salespersons high on customer orientation are likely to show higher concern for self and for others, but salespeople with sales orientation or having low customer orientation would exhibit high concern for self, but low concern for others. Therefore, it is also likely that a customer oriented salesperson is more likely to create value for customers, since s/he is likely to be more concerned for others (customers). Therefore, the following hypothesis is proposed.

H3: Customer orientation is positively associated with value co-creation.

\subsection{Communication culture}

Communication culture and structures - is the atmosphere within the organization that defines accepted communication behaviour which may facilitate or hinder the communication process [34-36]. The structures of communication and integration of knowledge are essential for enhancing the absorptive capacity [37]. Different authors regard the communication climate as an important factor impacting the communication process [38-39]. An open culture that views change as positive can facilitate communication and thus the learning process [38]. New knowledge creation requires routines that simplify and facilitate communication allows members of the firms to explore ways in which they can use existing knowledge or develop new knowledge [40]. They argue that structural, cognitive, behavioural and political barriers may stifle the effective sharing and integration of knowledge. Therefore, the following hypothesis is proposed. 
H4: Communication culture has a positive impact on value co-creation.

\section{Research methodology}

This study is based on an online questionnaire and selected sample e-commerce enterprises from the Information Management Association of R.O.C., International Service Industry Association of R.O.C. and International Trade Executives Association of R.O.C. from July 2013 to October 2014. The sampling frame is set to all the enterprises in the three agencies including CIS 400 companies, IMA 115 companies, ITEA 653 companies. In accordance with the principle of simple random sampling, the 1168 enterprises in sampling box will be carried out in order, and based on the stochastic indicator compiled by the US RAND in 1955, it begins to sample. According to the experience Sample size, Gaythinks that the sample is at least accounted for $10 \%$ of overall in the description research; Tabachnick, Fidell, Velicer, and Fava think that in the SEM analysis ,the tested samples had better be in more than 200. Therefore, the sample of study plan to determine the sample for 300 [41-42].

According to the sample plan, the sample company adopts method of questionnaire survey, and the sampling sample is $300 . \mathrm{We}$ design the questionnaire by considering related works and experts' views. After the first draft is completed, a pretest is undertaken with established leaders in outsourcing value co-creation in the IT industries to amend any part perceived to have a dubious presentation. Therefore, questionnaire recipients could understand the questions in the official survey, and a satisfactory completion of the questionnaire is ensured. All aspects are assessed using a five-point Likert scale from $1=$ 'strongly disagree' to $5=$ 'strongly agree'. We select a sample of 300 e-commerce enterprises; 293 ecommerce enterprises are removed from the initial sample because they have ceased trading, have a policy of not responding to surveys or requested their removal from the study. Sampling thus yields 207 useful questionnaires, a response valid rate of $97.67 \%$

The method chosen to analyze the data is the structural equations method, using the Partial Least Squares (PLS) technique. This technique is designed to reflect the theoretical and empirical characteristics of social sciences and behavioral characteristics, in which we often find theories with insufficient support or little information available. More specifically, we use Smart PLS 2.0 build M3.

\section{Research results}

Structural equation modeling (SEM) technique is used to test the hypotheses. SmartPLS software is used to analyze the data.We first examine the instrument (the measurement model) to assess reliability and validity before testing the structural model using the level of significance of the path coefficients and the variance explained (R square measures), as shown in Table 1.

Table 1. Descriptive statistics of the constructs.

\begin{tabular}{|c|c|c|c|c|c|c|}
\hline & AVE & $\mathrm{CR}$ & $\begin{array}{c}\mathrm{R} \\
\text { square }\end{array}$ & $\mathrm{CA}$ & Communality & Redundancy \\
\hline $\mathrm{CC}$ & 0.93 & 0.97 & & 0.96 & 0.93 & \\
\hline $\mathrm{CO}$ & 0.94 & 0.98 & & 0.97 & 0.94 & \\
\hline $\mathrm{CP}$ & 0.93 & 0.98 & & 0.97 & 0.93 & \\
\hline SPO & 0.89 & 0.96 & & 0.94 & 0.89 & \\
\hline $\mathrm{VC}$ & 0.87 & 0.95 & 0.89 & 0.93 & 0.87 & 0.64 \\
\hline \multicolumn{7}{|c|}{$\mathrm{CC}:$ Communication Culture } \\
\hline \multicolumn{7}{|c|}{$\mathrm{CO}:$ Customer Orientation } \\
\hline \multicolumn{7}{|c|}{$\mathrm{CP}$ :Customer Participation } \\
\hline \multicolumn{7}{|c|}{ SPO : Strategic Social Positioning } \\
\hline \multicolumn{7}{|c|}{ VC :Value Co-Creation } \\
\hline \multicolumn{7}{|c|}{ CR : Composite Reliability } \\
\hline \multicolumn{7}{|c|}{ CA : Cronbache Alpha } \\
\hline
\end{tabular}

\subsection{Measurement model}

We assess Cronbach's alpha, the composite reliability of constructs, average variance extracted (AVE), R square, and communality to test convergent validity. As shown in Table 1, the Cronbach's alpha values of all constructs range from 0.93 to 0.97 . Composite reliability values range from 0.95 to 0.98 and are above the benchmark value of 0.70 , suggesting acceptable degrees of internal consistency of each construct. AVE values range from 0.87 to 0.94 . Communality values range from 0.87 to 0.94 and are above the benchmark value of 0.50 . This will indicate that the model explains most of the variation for those variables.

The second criterion requires that the square root of AVE shall be greater than the correlations between the constructs. As seen in Table2, the square roots of AVEs (diagonal elements) are higher than the correlation between constructs (off-diagonal elements). The results support the discriminant validity of the scale used.

Table 2. Square root of AVES.

\begin{tabular}{|c|c|c|c|c|c|}
\hline & $\mathrm{CC}$ & $\mathrm{CO}$ & $\mathrm{CP}$ & $\mathrm{SPO}$ & $\mathrm{VC}$ \\
\hline $\mathrm{CC}$ & 0.96 & & & & \\
\hline $\mathrm{CO}$ & 0.92 & 0.97 & & & \\
\hline $\mathrm{CP}$ & 0.88 & 0.92 & 0.96 & & \\
\hline
\end{tabular}




\begin{tabular}{|c|c|c|c|c|c|}
\hline SPO & 0.83 & 0.82 & 0.80 & 0.94 & \\
\hline VC & 0.92 & 0.90 & 0.88 & 0.85 & 0.93 \\
\hline
\end{tabular}

\subsection{Structural model}

The structural model is assessed using the variance explained ( $\mathrm{R}$ square measures) and the level of significance of the $\beta$-Values and T values, as shown in Table1 and Table3. The $\mathrm{R}$ square results for the dependent variable, value co-creation $(\mathrm{R}$ square $=0.89$ ), is high. This suggests that the model has high explanatory power, explaining $89 \%$ of the variance in the dependent variable respectively.

Table 3. $\beta$ Value and $\mathrm{T}$ value.

\begin{tabular}{|c|c|c|}
\hline & $\beta$ Value & T Value \\
\hline $\mathrm{CP} \rightarrow \mathrm{VC}$ & 0.15 & 1.22 \\
\hline $\mathrm{SPO} \rightarrow \mathrm{VC}$ & 0.24 & 2.50 \\
\hline $\mathrm{CO} \rightarrow \mathrm{VC}$ & 0.19 & 1.24 \\
\hline $\mathrm{CC} \rightarrow \mathrm{VC}$ & 0.40 & 3.76 \\
\hline
\end{tabular}

The result for $\mathrm{H} 1$ linking strategic social positioning to value co-creation is supported $(\beta-$ Value $=0.24, \mathrm{~T}$ value $=2.50, \mathrm{p}<0.01$ ). Consistent with this, we find that customer participation to value co-creation $(\mathrm{H} 2)$ is supported $(\beta-$ Value $=0.15, \mathrm{~T}$ value $=1.22)$. Customer orientation to value co-creation (H3) is supported $(\beta$ Value $=0.19$, $\mathrm{T}$ value $=1.24$ ). Communication culture to value co-creation $(\mathrm{H} 4)$ is supported $(\beta$-Value $=0.48, \mathrm{~T}$ value $=3.76, \mathrm{p}<0.001)$. The main effect model is shown as Fig. 2.

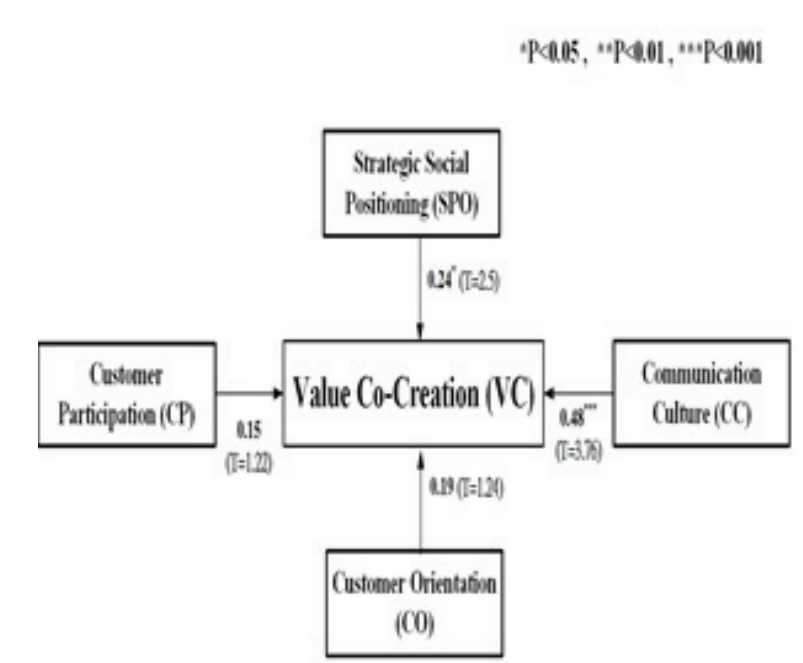

Fig.2. Research results.

\section{Discussion and contribution}

We formulate four hypotheses and conduct a field study to develop our understanding of how strategic social positioning, customer participation, customer orientation, and communication culture impact value co-creation in an organizational context. Our goal in this research is to document the association between IT outsourcing vendors and manufacturers. We find value co-creation has a strong impact on them.

The contributions of the paper are described as follows. First, this study contributes to manufacturers by integrating the perspective of economic and relational view in the study of the relational IT outsourcing vendors, which are not dealt with in previous studies. Second, this paper extends current research by highlighting the role of value-based relationships from the economic and relational view of manufacturers and IT outsourcing vendors.

In this study, we construct a new model to understand the factors influencing value co-creation between IT outsourcing vendors and manufacturers. Through studying Taiwan's IT outsourcing industries, we find that all factors modelled have an important effect on inter-organizational value creation and that there is a relation between vendors and manufacturers in these terms. We also find that these factors enhance the values of IT outsourcing vendors and manufacturers.

Future research will help identify the key factors that affect how consumers perceive and interpret website quality as a means for making product quality assessments when faced with high levels of information asymmetry. These findings provide a solid foundation for future investigations and practical insights for designing $\mathrm{B} 2 \mathrm{C}$ e-commerce websites. This study also makes a contribution by addressing cognitive lock-in (Johnson, Bellman, and Lohse, 2003) and its effects on 
perceived value and purchase intentions. These insights not only help researchers better understand how PPRs influence consumers' shopping behavior in electronic markets, but also provide guidelines for online retailers to better adjust their IT strategies to improve customer retention.

A growing concern among the organisations who are actively involved in Information Technology outsourcing is post-contract management and the ensuing development of what many practitioners and scholars have coined the 'outsourcing partnership'. Numerous corporations today are outsourcing specific information systems (IS) functions. Outsourcing of information system functions is growing rapidly. The diversity of these outsourcing arrangements goes well beyond that associated with the more traditional facilities management. The effect of service quality of the provider and the ability of companies to build a partnership on these relationships are hypothesized and studied. Increasing attention has been paid to building successful partnerships in information systems outsourcing.

A classic question faced by technology suppliers and buyers are whether to compete in the product markets or to cooperate through licensing. Understanding what paying customers want and how to create products and services they value is the cornerstone of any business model. Customer value can be examined at different levels. At a low level, customer value can be viewed as the attributes of a product that a customer perceives to receive value from. Facilitated by a wide array of technological advancements, interaction between customers and companies is taking new forms and shapes that go beyond traditional exchange. Companies are no longer perceived as sole goods providers, nor customers regarded merely as sources of money. Thus, both companies and customers are initiating new ways to support each other's value creation, that is, new value co-creation mechanisms are developed.

\section{References}

1. C. Koo, N. Chung, K. Nam, IJIM, 35 (1), pp.64-79, (2015)

2. J. Y. Qi, Q. X. Qu, Y. P. Zhou, ECRA, 13 (5), pp.295-304, (2014)

3. A. Padilla-Meléndez, A. Rosa del Águila-Obra, IJIM, 33 (5), 892-898, (2013)

4. M. Pagani, MISQ, 37 (2), 617-632, (2013)

5. M. J. Mol, J. Birkinshaw, OS, 35 (9), 1287-1312, (2014)

6. M. J. Mol, R. J. M. van Tulder, P. R. Beije, $I B R, 14$ (5), 599-617, (2005)

7. L. Ogiela, IJIM, 35 (2), 154-159, (2015)

8. M. R. Ogiela, IJIM, 34 (3), 317-318, (2014).
9. D. A. Mirchandani, A. L. Lederer, IJIM, 34 (5), 622$633,(2014)$

10. C. Woodard, R. N. Jason, F. T. Tschang, V. Sambamurthy, MISQ, 37(2), 537-564, (2013)

11. A. E. 1. Bharadwaj, O. A. Sawy, P. A. Pavlou, N. Venkatraman, MISQ, 37 (2), 471-482, (2013).

12. M. Oslo, The Measurement of Scientific and Technological Activities, A joint publication of OECD and Eurostat, (2005)

13. G. Battisti, P. Stoneman, BJM, 21 (1), 187-206, (2010)

14. F. Damanpour, R. M. Walker, C. N. Avellaneda, $J M S, 46$ (4), 650-675, (2009)

15. P. B. Goes, MISQ, 38 (1), piii-viii, (2014)

16. C. K. Prahalad, V. Ramaswamy, $S \& L, 36(3), 4-9$, (2004b)

17. P. P. Maglio, J. Spohrer, JMAS, 36(11), 18-20, (2008)

18. L.C Wu, L.-H. Wu, TJCIS, 51(2), 14-23, (2011)

19. B. Dong, K. R. Evans, S. Zou, JAMS, 36(1), 123 137, (2008)

20. D. A. Aaker, G. G. Shansby, BH, 25 (3), 56-62, (1982)

21. L. J. Harrison-Walker, JIBR, 10 (2), 135-147, (2011)

22. C.C.A. Claycomb, H. Lengnick, L.W. Inks, JBS, 18 (1), 47-69, (2001)

23. H. Zhang, Y. Lu, B. Wang, S. Wu, IM, 52 (4), 468482, (2015)

24. J. P. Foreyt, S. P. H. Walker, JADA, 98(10), 27-30, (1998)

25. R. L. Street, K. Edward, A. Robert, R. L. Bell, H. Kravitz, \& Paul, JGIM, 18 (8), 609-616,(2003)

26. R. Eisenberger, H. Robin, H. Steven, S. Debora, JAP, 71(3), 500-507, (1986)

27. J. H. Fleming, C. Coffman, J. K. Harter, HBR, 83(78), 106-114, (2005)

28. D. D. Gremler, K. P. Gwinner, JSR, 3(1), 82-104, (2000)

29. M. A. Jolson, JPSSM, 17(4), 75-88, (1997)

30. D. Korschun, C.B. Bhattacharya, S.D. Swain, $J M$, 78(3), 20-37, (2014)

31. E. G. Harris, T.L. Brown, C. John, J.C. Mowen, A. Artis, P \& M, 31(3), 171-183, (2014)

32. S. F. Slater, J. C. Narver, JM, 59 (3), 63-74, (1985)

33. R. Saxe, B. A. Weitz, JMR, 19 (3), 343-351, (1982) 
34. J. H. Adler, Washington: Brookings Institution, (1965)

35. S. L. Brown, K. M. Eisenhardt, $A S Q$, 42(1), 1-34, (1997)

36. J. Littler, CCC, 6(2), 227-243, (2013)

37. W. M. Cohen, D. A. Levinthal, ASQ, 35(1), 152-162, (1990)

38. N. S. Levinson, M. Asahi, OD, 24(2), 50-63, (1995)

39. W. Tsai, AMJ, 44 (5), 996-1005, (2001)

40. S.A. Zahra, G. George, AMR, 27 (2), 185-203, (2000)

41.B. G. Tabachnick, L. S. Fidell, Boston, Massachusetts: Allyn and Bacon, (2007)

42. W. F. Velicer, J. L. Fava, PM, 3 (2), 231-251, (1998) 